\title{
Starke Dualität bei verallgemeinerten Steuerproblemen
}

\section{S. Pickenhanin}

In dieser Arbeit wird eine verallgemeinerte Aufgabenstellung zu einem Variationsproblem in Parameterdarstellung unter Richtungs- und Gebietsbeschränkungen betrachtet und ein duales Problem konstruiert. Unter schwachen Voraussetzungen an das verallgemeinerte Problem wird starke Dualität gezeigt.

B әтой работе рассматривается обобщенная вариационная задача в параметрическом представлении с фазовыми ограничениями и ограничениями на управление. Построена двоиственпая аадача. При слабых предположениях доказана сильная двоиственность.

In this paper a generalized parametric variational problem with restrictions on the state domain and the control is considered and its dual problem constructed. Under weak assumptions, strong duality is shown between the primary and the dual problem.

\section{Einleitung}

Eine zentrale Stellung in der Theorie der mathematischen Optimierung nimmt die Dualität ein, durch die ein enger Zusammenhang zwischen einem gegebenen Problem und einem dazu dualen Problem hergestellt wird (vgl. z. B. [2, 4]).

Wir studieren ein Steuerproblem in der standardisierten Form eines Variationsproblems in Parameterdarstellung unter Richtungs- und Gebietsbeschränkungen:

$$
J(x)=\int_{0}^{T} r(x(t), \dot{x}(t)) d t \rightarrow \operatorname{Min} !
$$

bezüglich aller auf $[0, T]$ definierten vektorwertigen Funktionen $x=\left(x^{1}, \ldots, x^{n}\right)$, . $x \in X\left(x_{0}, x_{T}\right)$ mit

$$
\begin{aligned}
X\left(x_{0}, x_{T}\right)= & \left\{x \in W_{\infty}^{1, n}(0, T) \mid x(t) \in \bar{G} \text { auf }[0, T],\right. \\
& \dot{x}(t) \in U(x(t)) \text { fast überall auf }[0, T], \\
& \left.x(0)=x_{0}, \quad x(T)=x_{T}\right\}
\end{aligned}
$$

Dabei stellen wir folgende Grundvoraussetzungen an das Problem:

1. $G$ sei ein Lipschitzgebiet ${ }^{1}$ ) des $\mathbf{R}^{n}$.

2. $r$ sei eine stetige Funktion auf $\bar{G} \times \mathbf{R}^{n}$, positiv homogen 1. Grades bezüglich des zweiten Argumentes, und es gelte $r(\xi, v)>0$ für alle $v \neq 0, \xi \in \bar{G}$.

3. $U(\cdot)$ sei eine mengenwertige Abbildung von $\bar{G} \rightarrow \mathbf{R}^{n}$, deren Bilder $U(\xi)$ für alle $\xi \in \bar{G}$ abgeschlossene Kegel des $\mathbf{K}^{n}$ (mit der Spitze in Null) sind, außerdem sei $U_{1}(\cdot)$ mit $U_{1}(\xi)=\{v \in U(\xi)|| v \mid=1\}$ im Sinne von S. Rouswicz ([11], S. 171) bildstetig.

4. $X\left(x_{0}, \xi\right) \neq \varnothing$ für alle $\xi \in G$.

1) Im Sinne von S. Hrldebrandt (Math. Annalen 148 (1962), S. 230). 
Ein zu (1) duales Problem ist dann nach R. KLötzler [6] die Aufgabe

mit

$$
L(S):=S\left(x_{T}\right)-S\left(x_{0}\right) \rightarrow \operatorname{Max} ! \text { auf } \mathscr{A}(G)
$$

$$
\mathscr{A}(G):=\left\{S \in C^{1}(G) \mid \nabla S(\xi) \in \mathscr{F}(\xi) \text { für alle } \xi \in G\right\},
$$

wobei $\mathscr{F}(\xi)$ den sogenannten F'iguratrixkörper des Problems (1) darstellt,

$$
\mathscr{F}(\xi):=\left\{z \in \mathbf{R}^{n} \mid z^{T} \cdot v \leqq r(\xi, v) \text { für alle } v \in U(\xi)\right\} \text {. }
$$

Bémerkung: Wegen der Gutartigkeit von $G$ (Lipschitzgebiet) kann man den zulässigen Bereich $\mathscr{A}(G)$ auf $\overline{\mathscr{A}}(G)$ erweitern, ohne daß sich die Dualitätsaussage ändert, mit

$$
\mathscr{A}(G):=\left\{S \in W_{\infty}^{1}(\dot{G}) \mid \nabla S(\xi) \in \mathscr{F}(\xi) \text { für fast alle } \xi \in G\right\} .
$$

Von besonders großem Interesse ist nun die Frage nach starker Dualität zwischen (1) und (2), d. h. wann gilt

$$
\sup _{S \in \bar{\not}(G)} L(S)=\inf _{x \in X\left(x_{0}, x_{T}\right)} J(x) ?
$$

Stellt man die folgenden zusätzlichen Voraussetzungen an das Problem:

(A) $U(\xi)$ ist konvex für alle $\xi \in \bar{G}$,

(B) $r(\xi, \cdot)$ ist eine konvexe Funktion auf dem $\mathbf{h}^{\text {n }}$ für alle $\xi \in \bar{G}$, so kann man zwischen dem Primalproblem (1) und seinem dualen Problem (2) starke Dualität sichern, vgl. dazu R. KLöTzLer [7]. In dieser Arbeit soll nun ein zu (1) verallgemeinertes Problem (1) nach L. C. Young [14] und E. J. McShane [8] betrachtet und ein duales Problem konstruiert werden. Dabei stellt sich heraus, da $B$ (1) sowie das verallgemeinerte Problem (1) ein gleiches Dualproblem besitzen. Ohne Konvexitätsvoraussetzungen kann man zwischen (1) und (2) starke Dualität nachweisen. Aus der Kenntnis der Lösung des verallgemeinerten Problems, die unter sehr schwachen Voraussetzungen existiert, kann man mittels notwendiger Optimalitätsbedingungen dann auch auf die Lösung der ursprünglichen Aufgabe (1) schließen, vergleiche [9].

\section{Definition des verallgemeinerten Problems}

Wir führen zunächst den Begriff der verallgemeinerten Steuerung ein.

Definition: Eine Familie von Wahrscheinlichkeitsmaßen $\mu=\left\{\mu_{\ell}, t \in[0, T]\right\}$, die von einem Parameter $t \in[0, T]$ abhängen, heißt verallgemeinerte Steuerung, wenn gilt:

1. Für jede Funktion $g \in C\left(\mathbf{R}^{n}\right)$ sei die Funktion $h_{g}$ mit

$$
h_{0}(t)=\int_{U} g(v) d \mu_{t}(v)
$$

Lebesgue-meßbar.

2. Es existiere eine kompakte Menge $K \subset \mathbf{R}^{n}$, die nicht von $t$ abhängt, so daß die Maße $\mu_{t}$ für fast alle $t$ auf der Menge $K$ konzentriert sind.

Wir bezeichnen die Menge aller verallgemeinerten Steuerungen, die auf $U \subseteq \mathbf{R}^{n}$ konzentriert sind, mit $\mathscr{M}_{u}$.

Damit studieren wir folgende verallgemeinerte Aufgabenstellung:

$$
\bar{J}(x, \mu)=\int_{0}^{T} \int_{U} r(x(t), v) d \mu_{\ell}(v) d t \rightarrow \operatorname{Min} !
$$


bezüglich aller auf $[0, T]$ definierten Paare $(x, \mu) \in Y\left(x_{0}, x_{T}\right)$ mit

$$
\begin{aligned}
& Y\left(x_{0}, x_{T}\right)=\left\{(x, \mu) \mid \mu \in \mathscr{M}_{u} ; \quad \operatorname{supp} \mu_{t} \subseteq U(x(t)),\right. \\
& \dot{x}(t)=\int_{U} v d \mu_{t}(v) \quad \text { fast überall auf }[0, T] ; \\
& x(t) \in \bar{G} \quad \text { auf }[0, T] ; x\left(0=x_{0}, x(T)=x_{T}\right\}, \\
& U=\bigcup_{\xi \in \bar{G}} U(\xi) .
\end{aligned}
$$

\section{Einbettung ursprünglicher Steuerungen zum Problem (1)}

in die Menge der verallgemeinerten Steuerangen

Sei $u$ eine zulässige Steuerung zum Problem (1), dann ordnen wir dem Wert $u(t)$ der Steuerung $u$ in Punkt $t$ das positive Maß (Diracmaß) $\delta_{u(t)}$ zu, das im Punkt $u(t)$ $\epsilon U(x(t))$ konzentriert ist (supp $\left.\delta_{u(t)} \subseteq U(x(t))\right)$ und auf eine Funktion $g \in C\left(\mathbf{R}^{n}\right)$ gemäß der Formel

$$
\int_{U} g(v) d \delta_{u(t)}(v)=g(u(t))
$$

wirkt.

Weil die Steuerung $u$ fast überall beschränkt ist, besitzt

$$
\delta=\left\{\delta_{u(t)}, t \in[0, T]\right\}
$$

einen kompakten Träger, der nicht von $t$ abhängt und wegen der Meßbarkeit von $u$ ist die Funktion

$$
h_{o}(t)=g(u(t))
$$

Lebesgue-meßbar für jede stetige Funktion $g$, vergleiche z. B. [3], S. 287. Damit ist $\delta$ eine zulässige verallgemeinerte Steuerung zum Problem (i).

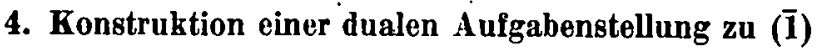

Wir führen zunächst folgende Bezeichnungen ein:

$H$ sei die Pontrjaginsche Funktion, definiert auf $\bar{G} \times \mathbf{R}^{n} \times \mathbf{R}^{n}$,

$$
H(\xi, v, y)=-r(\xi, v)+\sum_{i=1}^{n} y_{i} v_{i}
$$

und $\mathscr{H}$ die Hamiltonfunktion, definiert auf $\bar{G} \times \mathbf{R}^{n}$,

$$
\mathscr{H}(\xi, y)=\sup _{v \in U(\xi)} H(\xi, v, y)
$$

und zeigen folgenden Hilfssatz.

Hilfssatz 1: Für beliebige $\xi \in \mathbf{R}^{n}, y \in \mathbf{R}^{n}$ gill:

$$
\sup _{\mu \in P_{U(\xi)}} \int_{U} H(\xi, v, y) d \mu(v)=\sup _{v \in U(\xi)} H(\xi, v, y),
$$

wobei $P_{U(\xi)}$ die Menge der Wahrscheinlichkeitsmaße ist, die auf $U(\xi)$ konzentriert ist. 
Bewe is: 1. Die Ungleichung

$$
\sup _{\mu \in P U(\xi)} \int_{U(\xi)} H(\xi, v, y) d \mu(v) \geqq \sup _{v \in U(\xi)} H(\xi, v, y)
$$

erhält man, indem man das Supremum nur über alle Diracmaße erstreckt, die auf $U(\xi)$ konzentriert sind.

2. Um die umgekehrte Ungleichung zu zeigen, benutzen wir die Beziehung

$$
\int_{U(\xi)} f(\xi, v) d \mu(v) \in \operatorname{con} v\{f(\xi, v) \mid v \in U(\xi)\}
$$
für alle $\left.\mu \in P_{U(\xi)}, f \in C^{0, n+1}(G \times U)^{2}\right)$ und $f=\left(\begin{array}{l}r \\ v\end{array}\right)$, vergleiche [1]. Dann gilt für
alle $g \in \mathbf{R}^{n+1}, \mu \in P_{U(\xi)}$

$$
g^{T} \cdot \int_{U(\xi)} f(\xi, v) d \mu(v) \leqq \sup _{p \in c o n v\{f(\xi, v) \mid v \in U(\xi)\}} \hat{y}^{T} \cdot p
$$

Da für jeden $(n+1)$-dimensionalen Vektor $\hat{y}$ und jede Teilmenge $Q \subseteq \mathbf{R}^{n+1}$ die Gleichung

$$
\sup _{p \in \operatorname{con} v \bar{Q}} \hat{y}^{T} \cdot p=\sup _{p \in Q} \hat{y}^{T} \cdot p
$$

erfüllt ist, folgt

$$
\hat{y}^{T} \cdot \int_{U(\xi)} f(\xi, v) d \mu(v) \leqq \sup _{p \in c o n v(f(\xi, v) \mid v \in U(\xi))} \hat{y}^{T} \cdot p=\sup _{v \in U(\xi)} \hat{y}^{T} \cdot f(\xi, v) .
$$

$\hat{y}=\left(\begin{array}{c}-1 \\ y\end{array}\right)$ mit $y \in \mathbf{R}^{n}$ beliebig liefert die Behauptung

Die Konstruktion eines dualen Funktionals zu (I) verläuft analog zu der in [6] angegebenen.

Wir setzen $y_{i}=S_{\xi_{i}}(\xi)(i=1, \ldots, n)$ für eine beliebige Funktion $S \in C^{1}(G)$ und erhalten mittels (4), (5) und Hilfssatz 1 für einen zulässigen Prozeß $(x, \mu)$, der nach Grundvoraussetzung 4 existiert,

$$
\begin{aligned}
\bar{J}(x, \mu) & \left.=\int_{0}^{T}\left\{-H(x(t), v, \nabla S(x(t))) d \mu_{t}(v)+\nabla^{T} S(x(t)) \cdot \int_{v} v d \mu_{t}(v)\right\}\right\} d t \\
& \geqq \int_{0}^{T}\left\{-\mathscr{H}(x(t)), \nabla S(x(t))+\nabla^{T} S(x(t)) \dot{x}(t)\right\} d t .
\end{aligned}
$$

iIst

$$
\Lambda_{s}:=\sup _{\xi \in \bar{G}} \mathscr{H}(\xi, \nabla S(\xi)) \leqq 0
$$

für alle $S \in C^{1}(G)$, so folgt

$$
\bar{J}(x, \mu) \geqq \int_{0}^{T} \frac{d}{d t} S(x(t)) d t=S\left(x_{T}\right)-S\left(x_{0}\right) .
$$

Damit gewinnen wir als duale Aufgabe

$$
S\left(x_{T}\right)-S\left(x_{0}\right) \rightarrow \mathrm{Max} !
$$

2) $C^{0, n+1}(G \times U):$ Menge aller $(n+1)$-dimensionalen stetigen Vektorfunktionen auf $G \times U$. 
bezüglich aller $S \in \mathscr{A}(G)$ mit

$$
\mathscr{A}(G)=\left\{S \in C^{1}(G) \mid D S(\xi) \in \mathscr{F}(\xi) \text { für alle } \xi \in G\right\} .
$$

Die Aufgabe (2) erweist sich also als ein Dualproblem zu (1) ebenso wie zu (1). Wir erhalten damit folgende Ungleichung:

$$
\sup _{S \in \mathscr{A}(G)} L(S) \leqq \inf _{(x, \mu) \in Y\left(x_{0}, x_{T}\right)} \bar{J}(x, \mu) \leqq \inf _{x \in X\left(x_{*}, x_{T}\right)} J(x)
$$

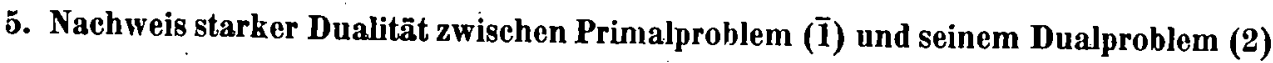

Wir stellen zunächst einige Eigenschaften verallgemeinerter Steuerungen zusammen, die wir zum Nachweis der starken Dualität benötigen.

Der Steuerbereich $U$ sei vom Zustand $\xi$ unabhängig, d. h. $U(\xi)=U$ für alle $\xi \in \bar{G}$, dann gilt:

A : Ist $(x, \mu) \in Y\left(x_{0}, x_{T}\right)$, dann existiert ein Paar $\left(x^{\prime}, \mu^{\prime}\right) \in P\left(x_{0}, x_{T}\right)$, so daß für den Träger der Maße $\mu_{\ell}^{\prime}$

$$
\operatorname{supp} \mu_{i}^{\prime}=\left\{v \in U|| v \mid=T^{-1} \mathscr{L}(\mu)\right\}
$$

fast überall auf $[0, T]$ mit

$$
\mathscr{L}(\mu):=\int_{0}^{T} \int_{U}|v| d \mu_{t}(v) d t
$$

gilt und

ist.

$$
\bar{J}(x, \mu)=\bar{J}\left(x^{\prime}, \mu^{\prime}\right)
$$

Bewe is: [8], Lemma 7.2, S. 526.

B: Die Menge der verallgemeinerten Steuerungen $\mu \in \mathscr{H}_{V}$, die auf einer kompakten Menge $V \subseteq U$ konzentriert sind, ist schwach*-kompakt, d.h. zu jeder Folge $\left\{u^{(n)}\right\}_{n-1}^{\infty}$ verallgemeinerter Steuerungen mit

$$
\text { supp } \mu_{t}^{(n)} \subseteq V \quad \text { für fast alle } t \in[0, T], \quad n=1,2, \ldots
$$

existiert eine Teilfolge $\left\{\mu^{\left(n^{\prime}\right\}}\right\}$, so daß für alle Funktionen $\left.g \in C_{0}\left(\mathbf{R}^{n+1}\right)^{3}\right)$ gilt: Es existiert eine verallgemeinerte Steuerung a mit

$$
\lim _{n^{\prime} \rightarrow \infty} \int_{0}^{T} \int_{V}^{T} g(t, v) d \mu_{t}^{\left(n^{\prime}\right)}(v) d t=\int_{0}^{T} \int_{V}^{T} g(t, v) d \mu_{t}(v) d t .
$$

Beweis: [1], Theorem 8.1, S. 140.

C: Sei $\left(x^{(n)}, \mu^{(n)}\right) \in Y\left(x_{0}, x_{T}\right)(n=1,2, \ldots)$ und $\left\{\mu^{(n)}\right\}$ konvergiere im Sinne der schwachen* Konvergenz gegen $A$, dann konvergiert die Folge der entsprechenden Trajektorien $x^{(n)}$ gleichmäßig gegen $\hat{x}$, mit

$$
\begin{aligned}
& \dot{\hat{x}}(t)=\int_{U} v d \mu_{l}(v) \quad \text { fast überall auf }[0, T], \\
& x(0)=x_{0}, \quad x(T)=x_{T} .
\end{aligned}
$$

3) $C_{0}\left(R^{n+1}\right)$ : Menge aller stetigen Funktionen auf $R^{n+1}$ mit kompaktem Träger. 
Weiter gilt

$$
\lim _{n \rightarrow \infty} \int_{0}^{T} \int_{U} r\left(x^{(n)}(t), v\right) d \mu_{t}^{(n)}(v) d t=\int_{0}^{T} \int_{U}^{T} r(\hat{x}(t), v) d \Lambda_{t}(v) d t .
$$

Beweis. [1], Theorem 4.4, S. 74 und Behauptung 6.2, S. 102.

Wir betrachten nun das Problem (1) ohne Steuerbeschränkungen, d. h. $U=U(\xi)$ $=\mathbf{R}^{n}$. Dann ist die Bellmansche Funktion

$$
S^{*}(\xi)=\inf _{x \in X\left(x_{0}, \xi\right)} \int_{0}^{T} r(x(t), \dot{x}(t)) d t
$$

zulässig für das duale Problem (d. h. $S^{*} \in \bar{A}(G)$ ) und liefert starke Dualität zwischen (1) und (2) und wegen Ungleichung (6) auch zwischen (1) und (2), vergleiche [7]. Dieses Ergebnis nutzen wir in weiteren, um mittels Penaltytechnik starke Dualität für dẹ Fall nachzuweisen, in dem $U(\xi)$ den in den Grundvoraussetzungen getroffenen Annahmen genügt.

Wir studieren folgende Schar von Ersatzaufgaben:

$$
\bar{J}_{k}(x, \mu)=\int_{0}^{T} \int_{\mathbf{R}^{\mathbf{n}}}\{r(x(t), v)+k \vartheta(x(t), v)\} d \mu_{t}(v) d t \rightarrow \operatorname{Min} !
$$

bezüglich aller auf $[0, T]$ definierten Paare $(x, \mu) \in Y_{0}\left(x_{0}, x_{T}\right)$ mit

$$
Y_{0}\left(x_{0}, x_{T}\right)=\left\{(x, \mu) \mid \mu \in \mathscr{M}_{\mathbf{R}^{n}} ; \dot{x}(t)=\int_{\mathbf{R}^{n}} v d \mu_{t}(v)\right.
$$

fast überall auf $[0, T], x(t) \in \bar{G}$ auf $\left.[0, T) ; x(0)=x_{0}, x(T)=x_{T}\right\}$.

Dabei ist

$$
\begin{aligned}
& \vartheta(\xi, e)=\inf _{z \in U_{1}(\xi)}|e-z| \\
& \text { mit e } \in E=\left\{y^{\circ} \in \mathbf{R}^{n}|| y \mid=1\right\} \text { und } \vartheta(\xi, v)=|v| \cdot \vartheta\left(\xi, \frac{v}{|v|}\right) \\
& \text { für } v \neq 0, \vartheta(\xi, 0)=0
\end{aligned}
$$

$(|\cdot|$ bezeichnet die euklidische Norm).

Hilfssatz 2: Die Funktion $\vartheta$ ist stetig auf $\bar{G} \times \mathbf{R}^{n}$ und positiv homogen ersten Grades im zweiten Argument.

Beweis: Wir zeigen die Stetigkeit von $\vartheta$ auf $\bar{G} \times E$. Seien $\varepsilon>0, \xi_{0} \in \bar{G}, e_{0} \in E$ beliebig vorgegeben. Nach Definition von $\vartheta$ gibt es ein $v \in U_{1}\left(\xi_{0}\right)$ mit

$$
\left|\underline{v}-e_{0}\right| \leqq \vartheta\left(\xi_{0}, e_{0}\right)+\varepsilon / 3 \text {. }
$$

Wegen der Unterhalbstetigkeit von $U_{1}(\cdot)$ in $\xi_{0}$ existiert ein $\delta\left(\varepsilon, \xi_{0}\right)$, so da $B$ für alle $\xi$ mit $\left|\xi-\xi_{0}\right|<\delta\left(\varepsilon, \xi_{0}\right)$ die Menge $U_{1}\left(\xi_{0}\right)$ in einer $\varepsilon / 3$-Umgebung von $U_{1}(\xi)$ enthalten ist. Folglich kann man ein $\bar{v} \in U_{1}(\xi)$ finden, für welches gilt $|\bar{v}-\underline{v}|<\varepsilon / 3$. Durch nochmalige Anwendung der Definition von $\vartheta$ gewinnen wir $\vartheta(\xi, e) \leqq|e-\bar{v}|$. Somit ist

d. h.

$$
\vartheta(\xi, e) \leqq\left|e-e_{0}\right|+\left|e_{0}-\underline{v}\right|+|\underline{v}-\bar{v}|<\varepsilon / 3+\vartheta\left(\xi_{0}, e_{0}\right)+\varepsilon / 3+\varepsilon / 3,
$$

$$
\vartheta(\xi, e)-\vartheta\left(\xi_{0}, e_{0}\right)<\varepsilon,
$$

für alle $\xi \in \bar{G}$ mit $\left|\xi-\xi_{0}\right|<\delta\left(\xi_{0}, \varepsilon\right)$ und für alle $e \in E$ mit $\left|e-e_{0}\right|<\varepsilon / 3$. 
Wir verwenden nun die Oberhalbstetigkeit der Abbildung $U_{1}(\cdot)$ in $\xi_{0}$. Fiir alle $\xi$ mit $\left|\xi-\xi_{0}\right|<\delta\left(\varepsilon, \xi_{0}\right)$ ist $U_{1}(\xi)$ in einer $\varepsilon / 3$-Umgebung von $U_{1}\left(\xi_{0}\right)$ enthalten. Wieder gilt nach Definition von $\vartheta$, daß ein $\underline{w} \in U_{1}(\xi)$ existiert, so da $\beta|\underline{w}-e| \leqq \vartheta(\xi, e)+\varepsilon / 3$ ist und es gibt ein $\bar{w} \in U_{1}\left(\xi_{0}\right)$ mit $|\underline{w}-\bar{w}|<\varepsilon / 3$. Damit ist

und

$$
\vartheta\left(\xi_{0}, e_{0}\right) \leqq\left|\bar{w}-e_{0}\right| \leqq\left|e_{0}-e\right|+|e-\underline{w}|+|\underline{w}-\bar{w}|
$$

$$
\vartheta\left(\xi_{0}, e_{0}\right)-\vartheta(\xi, e)<\varepsilon
$$

für alle $\xi \in \bar{G}$ mit $\left|\xi-\xi_{0}\right|<\delta\left(\varepsilon, \xi_{0}\right)$ und für alle $\dot{e} \in E$ mit $\left|e-e_{0}\right|<\varepsilon / 3$. (8) und (9) zusammen ergeben die Behauptung. Es ist leicht zu sehen, daß die Funktion $\vartheta$ dann auf $\bar{G} \times \mathbf{R}^{n}$ stetig ist. Die positive Homogenität ersten G'rades im zweiten Argument ergibt sich unmittelbar aus der Darstellung (7)

Wir wollen die Werte der Infima der Aufgabenschar $\left(\overline{1}_{k}\right)$ mit $\bar{I}_{k}\left(\dot{x}_{T}\right)$ bezeichnen. Die Folge der $\bar{I}_{k}\left(x_{T}\right)$ ist monoton wachsend und nach oben beschränkt, denn es ist für $k \stackrel{0}{=}, 1, \ldots$

$$
r(\xi, v)+k \vartheta(\xi, v) \leqq r(\xi, v)+(k+1) \vartheta(\xi, v)
$$

für alle $\xi \in \bar{G}$ und $v \in \mathbf{R}^{\mathbf{n}}$ und damit

$$
\begin{aligned}
& \bar{I}_{k}\left(x_{T}\right) \leqq \bar{I}_{k+1}\left(x_{T}\right), \\
& \bar{I}_{k}\left(x_{T}\right) \leqq \inf _{(x, \mu) \in Y\left(x_{0}, x_{T}\right)} \bar{J}_{k}(x, \mu) \leqq \inf _{x \in X\left(x_{0}, x_{T}\right)} J(x) \leqq J(\hat{x})
\end{aligned}
$$

für ein $\hat{x} \in X\left(x_{0}, x_{T}\right)$. Aus diesem Grunde existiert der Grenzwert

$$
\bar{I}^{*}\left(x_{T}\right):=\lim _{k \rightarrow \infty} \bar{I}_{k}\left(x_{T}\right)
$$

Zu den Problemen $\left(\overline{1}_{k}\right)$ können wir die entsprechenden Dualprobleme $\left(2_{k}\right)$ aufstellen:

$$
S_{k}\left(x_{T}\right)-S_{k}\left(x_{0}\right) \rightarrow \operatorname{Max} !
$$

bezüglich $S_{k} \in \bar{A}_{k}(G)$ mit

$$
\overline{\mathscr{A}}_{k}(G)=\left\{S_{k} \in W_{\infty}^{1}(G) \mid \nabla S_{k}(\xi) \in \mathscr{F}_{k}(\xi) \text { für fast alle } \xi \in G\right\}
$$

und

$$
\mathscr{F}_{k}(\xi)=\left\{z \in \mathbf{R}^{n} \mid z^{T} v \leqq r(\xi, v)+k \vartheta(\xi, v) \quad \text { für alle } v \in \mathbf{R}^{n}\right\}
$$

Zwischen $\left(2_{k}\right)$ und $\left(\overline{1}_{k}\right)$ liegt nach den am Anfang des Abschnittes $\mathbf{5}$ geführten Untersuchungen für den Fall, daß $U=\mathbf{R}^{n}$ ist, starke Dualität vor:

$$
\sup _{S_{k} \in \mathscr{B}_{k}(G)} L\left(S_{k}\right)=\sup _{S_{k} \in \mathscr{P}_{k}(G)}\left\{S_{k}\left(x_{T}\right)-S_{k}\left(x_{0}\right)\right\}=\inf _{(x, \mu) \in Y_{0}\left(x_{0} . x_{T}\right)} \bar{J}_{k}(x, \mu),
$$

d. h., zu jeder natürlichen Zahl $k$ kann man einen verallgemeinerten $\operatorname{Proze} B\left(x^{(k)}, \mu^{(k)}\right)$ $\epsilon Y_{0}\left(x_{0}, x_{T}\right)$ und eine Funktion $S_{k}^{*} \in S_{T_{k}}(G)$ finden, so daß

$$
\bar{J}_{k}\left(x^{(k)}, \mu^{(k)}\right)-\bar{I}_{k}\left(x_{T}\right) \leqq \frac{1}{k}
$$

und

$$
\bar{I}_{k}\left(x_{T}\right)=S_{k}^{*}\left(x_{T}\right)-S_{k}^{*}\left(x_{0}\right) .
$$

Ohne Beschränkung der Allgemeinheit nehmen wir an, daß der Prozeß $\left(x^{(k)}, \mu^{(k)}\right)$ die unter $\mathbf{A}$ dieses Abschnittes aufgeführte Eigenschaft habe, daß der Träger der 
Maße $\mu_{t}{ }^{(k)}$ für fast alle $t \in\left[0, T^{\prime}\right]$ die Menge

$$
\operatorname{supp} \mu_{i}^{(k)}=\left\{v \in \mathbf{R}^{n}|| v \mid=T^{-1} \mathscr{L}\left(\mu^{(k)}\right)\right\}
$$

ist. Wegen der Stetigkeit von $r$ und $r(\xi, v)>0$ für alle $\xi \in \bar{G}$ und $v \neq 0$ existiert das Minimum von $r$ auf $\bar{G} \times E$ :

$$
\operatorname{Min}_{\bar{G} \times \boldsymbol{E}} r(\xi, v)=m>0 .
$$

Dann folgt wegen der positiven Homogenität von $r(\xi, \cdot)$

$$
\begin{aligned}
\mathscr{L}\left(\mu^{(k)}\right) & =\int_{0}^{T} \int_{\mathbf{R}^{n}}|v| d \mu_{t}^{(k)}(v) d t \\
& \leqq m^{-1} \int_{0}^{T} \int_{\mathbf{R}^{n}} r\left(x^{(k)}(t), v\right) d \mu_{t}^{(k)}(v) d t \\
& \leqq m^{-1} \bar{J}_{0}\left(x^{(k)}, \mu^{(k)}\right) .
\end{aligned}
$$

Nach Ungleichung (10) ist

$$
0 \leqq \bar{J}_{0}\left(x^{(k)}, \mu^{(k)}\right) \leqq \bar{J}_{k}\left(x^{(k)}, \mu^{(k)}\right) \leqq \bar{I}_{k}\left(x_{T}\right)+\frac{1}{k} \leqq \bar{I}^{*}\left(x_{T}\right)+\frac{1}{k} .
$$

Folglich ist die verallgemeinerte Bogenlänge $\mathscr{L}\left(\mu^{(k)}\right)$ wegen

$$
\mathscr{L}\left(\mu^{(k)}\right) \leqq m^{-1}\left(\bar{I}^{*}\left(x_{T}\right)+1\right)
$$

für alle $k$ nach oben beschränkt. Nach Eigenschaft $\mathbf{A}$ und $\mathbf{B}$ kann man aus der Folge der $\left\{\mu^{(k)}\right\}$ eine Teilfolge $\left\{\mu^{\left(k^{\prime}\right)}\right\}$ auswählen, die im Sinne der schwachen* Konvergenz gegen $\mu^{*}$ konvergiert. Die Folge der entsprechenden Trajektorien $x^{\left(k^{\prime}\right)}$ konvergiert dann nach $\mathbf{C}$ gleichmäßig gegen $x^{*}$. Wegen der Beschränktheit von $r$ auf $\bar{G} \times E$ erhält man aus (12) für $k=k^{\prime}$ nach Division durch $k^{\prime}$ und anschließendem Grenzübergang $k^{\prime} \rightarrow \infty$

$$
\lim _{k^{\prime} \rightarrow \infty} \int_{0}^{T} \int_{\mathbf{R}^{n}} \vartheta\left(x^{\left(k^{\prime}\right)}(t), v\right) d \mu_{t}{ }^{\left(k^{\prime}\right)}(v) d t=0 .
$$

Wir verwenden die Aussage $\mathbf{C}$ dieses Abschnittes und erhalten

$$
\int_{0}^{T} \int_{\mathbf{R}^{n}} \vartheta\left(x^{*}(t), v\right) d \mu_{t}^{*}(v) d t=0 .
$$

Nach Definition von $\vartheta$ ist die verallgemeinerte Steuerung $\mu^{*}$ danit für fast alle $t$ auf $U\left(x^{*}(t)\right)$ konzentriert, d. h. $\left(x^{*}, \mu^{*}\right) \in Y\left(x_{0}, x_{T}\right)$. Wenn man noch einmal die Aussage $C$ berücksichtigt, erhält man mit (13)

$$
\lim _{k^{\prime} \rightarrow \infty} \bar{J}_{0}\left(x^{\left(k^{\prime}\right)}, \mu^{\left(k^{\prime}\right)}\right)=\bar{J}_{0}\left(x^{*}, \mu^{*}\right)=\bar{J}\left(x^{*}, \mu^{*}\right) .
$$

Weiterhin gewinnt man aus (10), (11), (12) und (14)

$$
\begin{aligned}
\bar{I}^{*}\left(x_{T}\right) & =\lim _{k^{\prime} \rightarrow \infty}\left\{S_{k^{\prime}}^{*}\left(x_{T}\right)-S_{k^{*}}^{*}\left(x_{0}\right)\right\}=\lim _{k^{\prime} \rightarrow \infty} \bar{J}_{k^{\prime}}\left(x^{\left(k^{\prime}\right)}, \mu^{\left(k^{\prime}\right)}\right) \\
& \geqq \lim _{k^{\prime} \rightarrow \infty} \bar{J}_{0}\left(x^{\left(k^{\prime}\right)}, \mu^{\left(k^{\prime}\right)}\right)=\bar{J}_{0}\left(x^{*}, \mu^{*}\right)=\bar{J}\left(x^{*}, \mu^{*}\right) .
\end{aligned}
$$


Würde in (15) $\bar{I}^{*}\left(x_{T}\right)>\bar{J}\left(x^{*}, \mu^{*}\right)$ gelten, so wäre.für $k^{\prime} \geqq k_{0}$ (hinreichend groß)

und

$$
S_{k^{*}}^{*}\left(x_{T}\right)-S_{k^{*}}^{*}\left(x_{0}\right)>\bar{J}\left(x^{*}, \mu^{*}\right)
$$

$$
\nabla S_{\boldsymbol{k}^{\prime}}^{*}(\xi) \in \mathscr{F}_{\boldsymbol{k}^{\prime}}(\xi) \subset \mathscr{F}(\xi)
$$

für alle $\xi \in G$, im Widerspruch zur starken Dualität zwischen den Problemen $\left(\overline{\mathbf{I}}_{k}\right)$ und $\left(2_{k}\right)$. Deshalb liegt zwischen (1) und (2) starke Dualität vor, $d . h$.

$$
\lim _{k \rightarrow \infty}\left\{S_{k^{*}}^{*}\left(x_{T}\right)-S_{k^{*}}^{*}\left(x_{0}\right)\right\}=\bar{J}\left(x^{*}, \mu^{*}\right)
$$

Anmerkung: Das inhomogene Steuerproblem

$$
F(x, u)=\int_{0}^{T} r(t, x(t), u(t)) d t \rightarrow \operatorname{Min} !
$$

bezüglich aller auf $[0 ; T]$ definierten vektorwertigen Funktionen $x=\left(x^{1}, \ldots, x^{n}\right)$ und Steuerungen $u$ mit $(x, u) \in X_{1}\left(x_{0}, x_{T}\right)$, wobei

$$
\begin{aligned}
X_{1}\left(x_{0}, x_{T}\right)= & \left\{(x, u) \mid u \in L_{\infty}^{m}(0, T) ; \quad \dot{x}(t)=g(t, x(t), u(t))\right. \\
& \text { und } u(t) \in U \subseteq \mathbf{R}^{m} \quad \text { fast überall auf }[0, T] ; \\
& \left.(t, x(t)) \in[0, T] \times \bar{G} ; \quad x(0)=x_{0}, \quad x(T)=x_{T}\right\}
\end{aligned}
$$

ist (hierbei hängt der Steuerbereich $U$ nicht von $(t, \xi) \in[0, T] \times \bar{G}$ ab), kann auf bckannte Weise, vergleiche $[10,12]$, auf ein Variationsproblem in Parameterdarstellung zurückgeführt werden, jedoch müssen zusätzliche Bedingungen an die Aufgabe gestellt werden, um die in den Grundvoraussetzungen geforderten Eigenschaften (z. B. die Stetigkeit des Integranden im Zielfunktional) zu erfüllen. In Untersuchungen von R. B. VINTER und R. M. Lewis [12, 13] werden solche Bedingungen angegeben, und es wird mit anderen Methoden eine Dualitätsaussage bewiesen, die der in dieser Arbeit gewonnenen entspricht.

\section{LITERATUR}

[1] Gamkrelidze, R. V.: Principles of Optimal Control Theory. Plenum Press: New York and London 1978.

[2] Golstern, E. G.: Dualitätstheorie in der nichtlinearen Optimierung und ihre Anwendung. Berlin 1975.

[3] Joffe, A. D., und V. M. Tichomirov: Theorie der Extremalaufgaben. Deutscher Verlag der Wissenschaften, Berlin 1979.

[4] Kuötzler, R.: Dualität und numerische Probleme der optimalen Steuerung. Seminarberichte der Humboldt-Universität Berlin 15 (1978), 65-75.

[5] Klötzler, R.: On a general conception of duality in optimal control. Proceedings of the conference EQUADIFF 4, Prague 1977, 189-196.

[6] KLötzler, R.: A generalization of the duality in optimal control and some numerical conclusions. Proceedings of the 8th. IFIP Conference on Optimization Techniques, Würzburg 1977, 313-320.

[7] KLötzLer, R.: Starke Dualität in der Steuertheorie. Math. Nachrichten 95 (1980), 253 bis 263.

[8] McShane, E. J.: Generalized curves. Duke Mathematical Journal 6 (1940), 513-536.

[9] McShane, E. J.: The calculus of variation from the beginning through optimal control theory. In: Optimal control and differential equations. Edited by: A. B. Schwarzkopf, Walter G. Kelley, and Stanley B. Elinson. All at the University of Oklahoma. Academic Press 1978, 3-49. 
[10] Rockafellar, R. T.: Conjugate convex functions in optimal control and the calculus of variations. Journal of Mathematical Analysis and Applications Vol. 32, No. 1 (1970), $174-222$.

[11] RoLewicz, S.: Funktionalanalysis und Steuertheoric. Berlin-Heidelberg-New York 1976.

[12] VINTER, R. B., and R. M. Lewis: The equivalence of the strong and weak formulation for certain problems in optimal control. SLAM J. on control and optimization. Vol. 16, No. 4 (1978), 546-570.

[13] VINTER, R. B., and R. M. LEwrs: A nessessary and sufficient condition for optimality of dynamic programming type, making no a priori assumptions on the controls. SIAM $J$. on control and optimization. Vol. 16 (1978), 571-583.

[14] Youna, L. C.: Lectures on the calculus of variations and optimal control theory. W. B. Saunders: Philadelphia 1969.

Manuskripteingang: 25.02.1981

\section{VERFASSER:}

Sabine Pickentain

Sektion Mathematik der Karl-Marx-Universität

DDR - 7010 Leipzig, Karl-Marx-Platz 10 